8th Alexander Friedmann International Seminar

on Gravitation and Cosmology

International Journal of Modern Physics: Conference Series

Vol. 3 (2011) 163-169

(C) World Scientific Publishing Company

DOI: $10.1142 /$ S2010194511001255

\title{
THE COSMOLOGICAL ORIGIN OF THE NAMBU-JONA-LASINIO MODEL
}

\author{
M. NOVELLO* \\ Instituto de Cosmologia Relatividade Astrofisica ICRA - CBPF \\ Rua Dr. Xavier Sigaud, 150, CEP 22290-180, Rio de Janeiro, Brazil \\ novello@cbpf.br
}

Received 27 June 2011

\begin{abstract}
Recently a mechanism to generate mass from gravitational interaction, based on Mach principle, according to which the inertia of a body is a property of matter as well as of the background provided by the rest-of-the-universe was presented in Refs. 1, 2. In these papers such an idea was realized for scalar and spinor fields treating the rest-of-theuniverse in its vacuum state. In the present paper, using an extended version of Mach principle, the same strategy will be applied to show how the Heisenberg-Nambu-JonaLasinio non-linear equation for fermions $\Psi$ arises as a consequence of the gravitational interaction of $\Psi$ with the rest-of-the-universe.
\end{abstract}

Keywords: Mach principle; Heisenberg-Nambu-Lasinio model.

PACS numbers: 04.20.Cv, 04.50.-h

\section{Introduction}

Although the theory of General Relativity may be understood as completely independent from the Machian idea that inertia of a body $\mathbb{A}$ is related to the global distribution of energy of all particles existing in the universe, we must recognize its historical value in the making the ideology that enabled Einstein to start his journey toward the construction of a theory of gravitation. ${ }^{3,4}$

During the 20th century, the idea of associating the dependence of local characteristics of matter with the global state of the universe came up now and then but without producing any reliable mechanism that could support such proposal.

Even the concept of mass - that pervades all gravitational processes - did not find a realization of such dependence on global structure of the universe. On the contrary, the most efficient mechanism and one that has performed an important role in the field of microphysics came from elsewhere, namely the attempt to unify forces of a non-gravitational character, such as long-range electrodynamics with decaying phenomena described by weak interaction. Indeed, the Higgs model produced an

* M. Novello is Cesare Lattes ICRANet Professor. 
efficient scenario for generating mass to the vector bosons ${ }^{5}$ that goes in the opposite direction of the proposal of Mach. This mechanism starts with the transformation of a global symmetry into a local one and the corresponding presence of vector gauge fields. Then, a particular form of the dynamics represented by $L_{\text {int }}(\varphi)$ of self-interaction of an associated scalar field in its fundamental state represented by an energy-momentum tensor given by $T_{\mu \nu}=L_{i n t}\left(\varphi_{0}\right) g_{\mu \nu}$ appears as the vehicle which provides mass to the gauge fields.

Recently a new mechanism for generation of mass that is a realization of Mach's idea was proposed. ${ }^{1}$ The strategy used in that paper is the following. We start with a theory of a massless scalar field in Ref. 1 (and for fermions in Ref. 2) coupled non-minimally to gravity through an interacting Lagrangian of the form $V(\varphi) R$. The distribution of the vacuum energy of the rest-of-the-universe is represented by a cosmological term $\Lambda$. The effect of $\Lambda$ by the intermediary of the dynamics of the metric of space-time in the realm of General Relativity is precisely to give mass to the field. Although this mass depends on the cosmological constant, its value cannot be obtained a priori.

In the present paper we apply this strategy in order to generalize Mach's idea, and following Dirac, Hoyle and others, to produce a mechanism that can transform the vague idea according to which local properties may depend on the universe's global characteristics, into an efficient process.

The first question we have to face concerns the choice of the elementary process. There have been many discussions in the scientific literature in the last decades related to the cosmic dependence of the fundamental interactions. We will do not analyze any of these here. On the contrary, we will concentrate on a specific selfinteraction of an elementary field and show that its correspondent dynamics is a consequence of a dynamical cosmological process. That is, to show that dynamics of elementary fields in the realm of microphysics, may depend on the global structure of the universe.

There is no better way than start our analysis with the fundamental theory proposed by Nambu and Jona-Lasinio concerning a dynamical model of elementary particles. ${ }^{6}$ Since the original paper until today hundreds of papers devoted to the NJL model were published. ${ }^{7}$ For our purpose here it is enough to analyze the nonlinear equation of motion that they used in their original paper as the basis of their theory which is

$$
i \gamma^{\mu} \nabla_{\mu} \Psi-2 s\left(A+i B \gamma^{5}\right) \Psi=0
$$

where $A=\bar{\Psi} \Psi$ and $B=i \bar{\Psi} \gamma^{5} \Psi$. This equation, as remarked by these authors, was proposed earlier by Heisenberg ${ }^{8}$ although in a quite different context. We will not enter in the analysis of the theory that follows from this dynamics. Our question here is just this: is it possible to produce a model such that HNJL (Heisenberg-NambuJona-Lasinio) equation for spinor field becomes a consequence of the gravitational interaction of a free massless Dirac field with the rest-of-the-universe? I claim that the answer is yes. This article was written to prove this. 


\section{The Cosmological Influence on the Microphysical World: The Case of Chiral-Invariant Heisenberg-Nambu-Jona-Lasinio Dynamics}

We take Mach's principle as the statement according to which the inertial properties of a body $\mathbb{A}$ are determined by the energy-momentum throughout all space. The description of such universal state that takes into account the whole contribution of the rest-of-the-universe onto $\mathbb{A}$ is the most homogeneous one and is related to what Einstein attributed to the cosmological constant or, in modern language, the vacuum of all remaining bodies. We follow a similar procedure here and will consider that the Extended Mach Principle (EMP) means that the influence of the rest-ofthe-universe on any elementary field in microphysics can be described through the identification of the energy-momentum distribution of all remaining bodies under the form

$$
T_{\mu \nu}^{U}=\Lambda g_{\mu \nu}
$$

Let us consider non-minimal coupling with gravity. In the framework of General Relativity we set the dynamics of a fermion field $\Psi$ coupled non-minimally with gravity to be given by the Lagrangian (we are using units were $\hbar=c=1$ )

$$
L=L_{D}+\frac{1}{\kappa} R+V(X) R-\frac{1}{\kappa} \Lambda+L_{C T}
$$

where

$$
L_{D} \equiv \frac{i}{2} \bar{\Psi} \gamma^{\mu} \nabla_{\mu} \Psi-\frac{i}{2} \nabla_{\mu} \bar{\Psi} \gamma^{\mu} \Psi
$$

The non-minimal coupling of the spinor field with gravity is contained in the term $V(X)$ and depends on the scalar $X$ defined by

$$
X=A^{2}+B^{2} \text {. }
$$

We note that we can write, in an equivalent way,

$$
X=J_{\mu} J^{\mu}
$$

where $J^{\mu}=\bar{\Psi} \gamma^{\mu} \Psi$. This quantity $X$ is chiral invariant, once it is invariant under the map

$$
\Psi^{\prime}=\gamma^{5} \Psi
$$

Indeed, from this $\gamma^{5}$ transformation, it follows

$$
A^{\prime}=-A, B^{\prime}=-B ; \text { then, } X^{\prime}=X
$$

The case in which the theory breaks chiral invariance the potential $V$ depends only on the invariant $A$ - and it is the road to the appearance of a mass. ${ }^{2}$ Here 
we start from the beginning with a chiral invariant theory. For the time being the dependence of $V$ on $X$ is not fixed. We have added $L_{C T}$ to counter-balance the terms of the form $\partial_{\lambda} X \partial^{\lambda} X$ and $\square X$ that appear due to the gravitational interaction. The most general form of this counter-term is

$$
L_{C T}=H(X) \partial_{\mu} X \partial^{\mu} X .
$$

We shall see that $H$ depends on $V$ and if we set $V=0$ then $H$ vanishes. This dynamics represents a massless spinor field coupled non-minimally with gravity. The cosmological constant is added by the reasons presented above and as we shall see it represents the influence of the rest-of-the-universe on $\Psi$.

Independent variation of $\Psi$ and $g_{\mu \nu}$ yields

$$
i \gamma^{\mu} \nabla_{\mu} \Psi+\Omega\left(A+i B \gamma^{5}\right) \Psi=0,
$$

where

$$
\begin{gathered}
\Omega \equiv 2 R V^{\prime}-2 H^{\prime} \partial_{\mu} X \partial^{\mu} X-4 H \square X \\
\alpha_{0}\left(R_{\mu \nu}-\frac{1}{2} R g_{\mu \nu}\right)=-T_{\mu \nu} .
\end{gathered}
$$

Here, we set $\alpha_{0} \equiv 2 / \kappa$ and $V^{\prime} \equiv \partial V / \partial X$. The energy-momentum tensor defined by

$$
T_{\mu \nu}=\frac{2}{\sqrt{-g}} \frac{\delta(\sqrt{-g} L)}{\delta g^{\mu \nu}}
$$

is given by

$$
\begin{aligned}
T_{\mu \nu} & =\frac{i}{4} \bar{\Psi} \gamma_{(\mu} \nabla_{\nu)} \Psi-\frac{i}{4} \nabla_{(\mu} \bar{\Psi} \gamma_{\mu)} \Psi \\
& +2 V\left(R_{\mu \nu}-\frac{1}{2} R g_{\mu \nu}\right)+2 \nabla_{\mu} \nabla_{\nu} V-2 \square V g_{\mu \nu} \\
& +2 H \partial_{\mu} X \partial_{\nu} X-H \partial_{\lambda} X \partial^{\lambda} X g_{\mu \nu}+\frac{\alpha_{0}}{2} \Lambda g_{\mu \nu}
\end{aligned}
$$

Taking the trace of Eq. (5), after some simplification and using

$$
\square V=V^{\prime} \square X+V^{\prime \prime} \partial_{\mu} X \partial^{\mu} X
$$

it follows

$$
\begin{aligned}
\left(\alpha_{0}+2 V+2 V^{\prime} X\right) R & =\left(4 H X-6 V^{\prime}\right) \square X \\
& +\left(2 H^{\prime} X-6 V^{\prime \prime}-2 H\right) \partial_{\alpha} X \partial^{\alpha} X \\
& +2 \alpha_{0} \Lambda .
\end{aligned}
$$

Then

$$
\Omega=\left(\mathbb{M} \square X+\mathbb{N} \partial_{\mu} X \partial^{\mu} X\right)+\frac{4 \alpha_{0} \Lambda V^{\prime}}{\alpha_{0}+2 V+2 V^{\prime} X},
$$

where

$$
\mathbb{M}=\frac{2 V^{\prime}\left(4 H X-6 V^{\prime}\right)}{\alpha_{0}+2 V+2 V^{\prime} X}-4 H
$$




$$
\mathbb{N}=\frac{2 V^{\prime}\left(2 X H^{\prime}-6 V^{\prime \prime}-2 H\right)}{\alpha_{0}+2 V+2 V^{\prime} X}-2 H^{\prime} .
$$

Defining $\Delta \equiv \alpha_{0}+2 V+2 V^{\prime} X$ we re-write $\mathbb{M}$ and $\mathbb{N}$ as

$$
\begin{aligned}
& \mathbb{M}=-\frac{4}{\Delta}\left(3 V^{\prime 2}+H\left(\alpha_{0}+2 V\right)\right), \\
& \mathbb{N}=-\frac{2}{\Delta}\left(3 V^{\prime 2}+H\left(\alpha_{0}+2 V\right)\right)^{\prime} .
\end{aligned}
$$

Inserting this result on Eq. (4) yields

$$
i \gamma^{\mu} \nabla_{\mu} \Psi+\left(\mathbb{M} \square X+\mathbb{N} \partial_{\lambda} X \partial^{\lambda} X\right) \Psi+\mathbb{Z}\left(A+i B \gamma^{5}\right) \Psi=0,
$$

where

$$
\mathbb{Z}=\frac{4 \alpha_{0} \Lambda V^{\prime}}{\alpha_{0}+2 V+2 V^{\prime} X}
$$

At this stage it is worth to select among all possible candidates of $V$ and $H$ particular ones that makes the factor on the gradient and on $\square$ of the field to disappear from Eq. (10).

The simplest way is to set $\mathbb{M}=\mathbb{N}=0$, which is satisfied if

$$
H=-\frac{3 V^{\prime 2}}{\alpha_{0}+2 V} .
$$

Imposing that $\mathbb{Z}$ must reduce to a constant we obtain

$$
V=\frac{1}{\kappa}\left[\frac{1}{1+\beta X}-1\right] \text {. }
$$

As a consequence of this,

$$
H=-\frac{3 \beta^{2}}{2 \kappa} \frac{1}{(1+\beta X)^{3}},
$$

where $\beta$ is a constant. Note that $V$ vanishes when $\beta=0$, and as a consequence, $H$ vanishes too.

The equation for the spinor becomes

$$
i \gamma^{\mu} \nabla_{\mu} \Psi-2 s\left(A+i B \gamma^{5}\right) \Psi=0
$$

where

$$
s=\frac{2 \beta \Lambda}{\kappa(\hbar c)} .
$$

Thus as a result of the above process the field satisfies Heisenberg-Nambu-JonaLasinio equation of motion. This is possible due to the influence of the rest-of-theUniverse on $\Psi$. If $\Lambda$ vanishes then the constant of the self-interaction of $\Psi$ vanishes. This is precisely what we envisaged to obtain: the net effect of the non-minimal coupling of gravity with the spinor field corresponds to a specific self-interaction. The HNJL equation of motion appears only if we take into account the existence 
of all remaining bodies in the universe - represented by the cosmological constant - in the state in which all existing matter is on the corresponding vacuum.

The various steps of our mechanism can be synthesized as follows:

- The dynamics of a massles spinor field $\Psi$ is described by the Lagrangian

$$
L_{D}=\frac{i}{2} \bar{\Psi} \gamma^{\mu} \nabla_{\mu} \Psi-\frac{i}{2} \nabla_{\mu} \bar{\Psi} \gamma^{\mu} \Psi
$$

- Gravity is described in General Relativity by the scalar of curvature

$$
L_{E}=R
$$

- The field interacts with gravity in a non-minimal way described by the term

$$
L_{\text {int }}=V(X) R,
$$

where $X=A^{2}+B^{2}$ and $A=\bar{\Psi} \Psi$ and $B=i \bar{\Psi} \gamma^{5} \Psi$

- The action of the rest-of-the-universe on the spinor field, through the gravitational intermediary, is contained in the form of an additional constant term on the Lagrangian noted as $\Lambda$;

- A counter-term depending on the invariant $X$ is introduced to kill extra terms coming from gravitational interaction;

- As a result of this process, after choosing $V$ and $H$ the field acquires a self-interaction term and its equation of motion is precisely HeisenbergNambu-Jona-Lasinio described as

$$
i \gamma^{\mu} \nabla_{\mu} \Psi-2 s\left(A+i B \gamma^{5}\right) \Psi=0,
$$

where $s$ is given by Eq. (14) and is zero only if the cosmological constant vanishes.

It is not hard to envisage others situations in which the above mechanism can be further applied. Then the question appears: how far one can extend this argument? We will come back to this in a future work.

\section{Conclusion}

In this paper we considered the influence of all the material content of the universe on a fermionic field when this content is in two possible states: in one case its energy distribution is zero; in another case it is in a vacuum state represented by the homogeneous distribution $T_{\mu \nu}=\Lambda g_{\mu \nu}$. In the first case the dynamics of the field is independent of the global properties of the universe and is described by the massless Dirac equation

$$
i \gamma^{\mu} \nabla_{\mu} \Psi=0
$$

In the second case, the rest-of-the-universe induces on field $\Psi$ the HeisenbergNambu-Jona-Lasinio non-linear dynamics

$$
i \gamma^{\mu} \nabla_{\mu} \Psi-2 s\left(A+i B \gamma^{5}\right) \Psi=0 .
$$


Thus, the scenario presented in this paper led us to consider the idea that microphysics can depend strongly on the global structure of the universe.

\section{Acknowledgments}

I would like to thank FINEP, CNPq and FAPERJ for financial support. I thank Ugo Moschella for conversations on the Nambu-Jona-Lasinio paper.

\section{References}

1. M. Novello, A mechanism to generate mass (submitted paper).

2. M. Novello, A mechanism to generate mass: the case of fermions, arXiv:1003.5126v1.

3. V. P. Vizgin and Ya. A. Smorodinskii, Sov. Phys. Uspekni 22, 489 (1979).

4. R. H. Dicke, Mach's Principle and a Relativistic Theory of Gravity, in Relativity, Groups and Topology (Gordon and Breach Publishers, Les Houches, 1964).

5. F. Halzen and A. D. Martin, Quarks and Leptons: An Introductory Course in Modern Particle Physics (John Wiley and Sons, New York, 1964).

6. Y. Nambu and G. Jona-Lasinio, Phys. Rev. 122, 345 (1961).

7. M. K. Volkov and A. E. Radzhabov, Forty-fifth anniversary of the Nambu-JonaLasinio model, arXiv:hep-ph/0508263.

8. W. Heisenberg, Z. Naturforsch. 14, 441 (1959) and earlier papers quoted there. 